\title{
Selecting the Links in BisoNets Generated from Document Collections
}

\author{
Marc Segond and Christian Borgelt \\ European Center for Soft Computing, \\ Calle Gonzalo Gutiérrez Quirós s/n, E-33600 Mieres (Asturias), Spain \\ \{marc.segond, christian.borgelt\}@softcomputing.es
}

\begin{abstract}
According to Koestler, the notion of a bisociation denotes a connection between pieces of information from habitually separated domains or categories. In this chapter, we consider a methodology to find such bisociations using a BisoNet as a representation of knowledge. In a first step, we consider how to create BisoNets from several textual databases taken from different domains using simple text-mining techniques. To achieve this, we introduce a procedure to link nodes of a BisoNet and to endow such links with weights, which is based on a new measure for comparing text frequency vectors. In a second step, we try to rediscover known bisociations, which were originally found by a human domain expert, namely indirect relations between migraine and magnesium as they are hidden in medical research articles published before 1987. We observe that these bisociations are easily rediscovered by simply following the strongest links.
\end{abstract}

\section{Introduction}

The concept of association is at the heart of many of today's powerful ICT technologies such as information retrieval and data mining. These technologies typically employ "association by similarity or co-occurrence" in order to discover new information that is relevant to the evidence already known to a user.

However, domains that are characterized by the need to develop innovative solutions require a form of creative information discovery from increasingly complex, heterogeneous and geographically distributed information sources. These domains, including design and engineering (drugs, materials, processes, devices), areas involving art (fashion and entertainment), and scientific discovery disciplines, require a different ICT paradigm that can help users to uncover, select, re-shuffle, and combine diverse contents to synthesize new features and properties leading to creative solutions. People working in these areas employ creative thinking to connect seemingly unrelated information, for example, by using metaphors or analogical reasoning. These modes of thinking allow the mixing of conceptual categories and contexts, which are normally separated. The functional basis for these modes is a mechanism called bisociation (see [1]).

According to Arthur Koestler, who coined this term, bisociation means to join unrelated, and often even conflicting, information in a new way. It means 
being "double minded" or able to think on more than one plane of thought simultaneously. Similarly, Frank Barron [2] says that the ability to tolerate chaos or seemingly opposite information is characteristic of creative individuals.

Several famous scientific discoveries are good examples of bisociations, for instance Isaac Newton's theory of gravitation and James C. Maxwell's theory of electromagnetic waves. Before Newton, a clear distinction was made between sub-lunar (below the moon) and super-lunar physics (above the moon), since it was commonly believed that these two spheres where governed by entirely different sets of physical laws. Newton's insight that the trajectories of planets and comets can be interpreted in the same way as the course of a falling body joined these habitually separated domains. Maxwell, by realizing that light is an electromagnetic wave, joined the domains of optics and electromagnetism, which, at his time, were also treated as unrelated areas of physical phenomena.

Although the concept of bisociation is frequently discussed in cognitive science, psychology and related areas (see, for example, 123 ), there does not seem to exist a serious attempt at trying to formalize and computerize this concept. In terms of ICT implementations, much more widely researched areas include association rule learning (for instance, [4]), analogical reasoning (for example, [5]6]), metaphoric reasoning (for example, 7]), and related areas such as case-based reasoning (for instance, [8]) and hybrid approaches (for example, [9]).

In order to fill this gap in current research efforts, the BISON project 11 was created. This project focuses on a knowledge representation approach with the help of networks of named entities, in which bisociations may be revealed by link discovery and graph mining methods, but also by computer-aided interactive navigation. In this chapter we report first results obtained in this project.

The rest of this chapter is structured as follows: in Section 2 we provide a definition of the core notion of a bisociation, which guides our considerations. Based on this definition, we justify why a network representation-a so-called BisoNet - is a proper basis for computer-aided bisociation discovery. Methods for generating BisoNets from heterogeneous data sources are discussed in Section 3 , including procedures for selecting the named entities that form its nodes and principles for linking them based on the information extracted from the data sources. In particular, we present a new measure for the strength of a link between concepts that are derived from textual data. Such link weights are important in order to assess the strength of indirect connections like bisociations.

Afterwards, in Section 5 we report results on a benchmark data set (consisting of titles and abstracts of medical research articles), in which a human domain expert already discovered hidden bisociations. By showing that with our system we can create a plausible BisoNet from this data source, in which we can rediscover these bisociations, we provide evidence that the computer-aided search for bisociations is a highly promising technology.

Finally, in Section [ 6 we draw conclusions from our discussion.

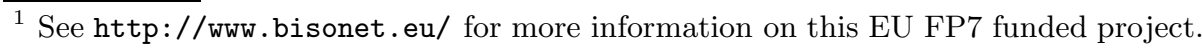




\section{Reminder: Bisociation and BisoNets}

Since the core notion of our efforts is bisociation, we start by trying to provide a sufficiently clear definition, which can guide us in our attempts to create a system able to support a user in finding bisociations. A first definition within the BISON project 2 characterizes bisociation as follows:

A bisociation is a link $L$ that connects two domains $D_{1}$ and $D_{2}$ that are unconnected given a specific context or view $V$ by which the domains are defined. The link $L$ is defined by a connection between two concepts $c_{1}$ and $c_{2}$ of the respective domains.

Although the focus on a connection between two habitually (that is, in the context a user is working in) separated domains is understandable, this definition seems somewhat too narrow. Linking two concepts from the same domain, which are unconnected within the domain, but become connected by employing indirect relations that pass through another domain, may just as well be seen as bisociations. The principle should rather be that the connection is not fully contained in one domain (which would merely be an association), but needs access to a separate domain. Taking this into account, we generalize the definition:

A bisociation is a link $L$ between two concepts $c_{1}$ and $c_{2}$, which are unconnected given a specific context or view $V$. The concepts $c_{1}$ and $c_{2}$ may be unconnected, because they reside in different domains $D_{1}$ and $D_{2}$ (which are seen as unrelated in the view $V$ ), or because they reside in the same domain $D_{1}$, in which they are unconnected, and their relation is revealed only through a bridging concept $c_{3}$ residing in some other domain $D_{2}$ (which is not considered in the view $V$ ).

In both of these characterizations we define domains formally as sets of concepts. Note that a bridging concept $c_{3}$ is usually also required if the two concepts $c_{1}$ and $c_{2}$ reside in different domains, since direct connections between them, even if they cross the border between two domains, can be expected to be known and thus will not be interesting or relevant for a user.

Starting from the above characterization of bisociation, a network representation, called a BisoNet, of the available knowledge suggests itself: each concept (or, more generally, any named entity) gives rise to a node. Concepts that are associated (according to the classical paradigm of similarity or co-occurrence) are connected by an edge. Bisociations are then indirect connections (technically paths) between concepts, which cross the border between two domains.

Note that this fits both forms of bisociations outlined above. If the concepts $c_{1}$ and $c_{2}$ reside in different domains, the boundary between these two domains necessarily has to be crossed. If they reside in the same domain, one first has to leave this domain and then come back in order to find a bisociation.

${ }^{2}$ See http://www.inf.uni-konstanz.de/bisonwiki/index.php5, which, however, is not publicly accessible at this time. 


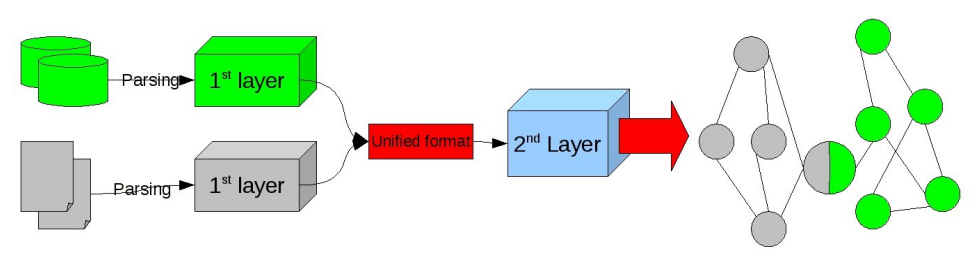

Fig. 1. Illustration of the structure of the BisoNet generator

\section{BisoNet Generation}

A system for generating BisoNets requires three ingredients: (1) A component to access the original, usually heterogeneous data sources. In order to cope with different data formats, we suggest, in Section [3.1] a two-layer architecture. (2) A method for choosing the named entities that are to form the nodes of the BisoNet. Here we rely on standard keyword extraction techniques, as discussed in Section 3.2. (3) A procedure for linking the nodes of a BisoNet and for endowing them with weights that indicate the association strength. For this we suggest, in Section 4, a new association measure for keywords.

\subsection{Data Access and Pre-processing}

As explained above, a BisoNet is a network that promises to contain bisociations. In order to generate such networks, we first have to consider two things: we must be able to read different and heterogeneous data sources, and we have to be able to merge the information derived from them in one BisoNet. Data sources can be databases (relational or of any other type), text collections, raw text, or any data that provide information about a domain. Due to the wide variety of formats a data source can have, the choice we made here is not to provide an interface of maximal flexibility that can be made to read any data source type, but to structure our creation framework into two separate steps.

In the first step, we directly accesses the data source and therefore a parser has to be newly developed for or at least adapted to the specific format of the data source. The second step is actual the BisoNet generation part. It takes its information from the first step, always in the same format, and therefore can generate a BisoNet from any data source, as far as it is parsed and exported in the form provided by the first step process (see Figure 1 for a sketch).

The way data should be provided to the second layer is fairly simple, because in this chapter we confine our considerations to textual data. As a consequence, the second layer creates nodes from data that are passed as records containing textual fields. These textual fields can contain, for now, either words or authors names. This procedure and data format is well adapted to textual databases or text collections, but is meant to evolve in future development in order to be able to take other types of data sources into account. However, since most of the data sources that we have used so far were textual data sources, this protocol seems simple and efficient. Future extensions could consist in including raw data 
fields (for example, to handle images), and will then require an adaptation of the second layer to be able to create nodes from other objects than textual data.

The second layer builds a BisoNet by extracting keywords using standard text mining techniques such as stop word removal and stemming (see [10]). The extracted keywords are weighted by their TFIDF (Text Frequency - Inverse Document Frequency) value (see [11]), thus allowing us to apply a (user-defined) threshold in order to filter the most important keywords, as will be detailed in Section 3.2. Links between nodes are created according to the presence of co-occurrences of the corresponding keywords in the same documents, and are weighted using a similarity measure adapted to the specific requirements of our case, which will be presented in Section 4. In the case that author lists are provided with each text string, extracted keywords are also linked to the related authors. These links are weighted according to the number of times a keyword occurs in a given author's work.

\subsection{Creating Nodes}

In our BisoNets nodes represent concepts. As we only talk about textual databases, we made the choice to characterize concepts by keywords that are extracted from the textual records taken from the data sources. In the second layer of our framework, each textual record $j$ is processed with a stop word removal algorithm. Then the text frequency values are computed for each remaining term $i$ as follows: $\mathrm{tf}_{i, j}=\frac{n_{i, j}}{\sum_{k} n_{k, j}}$, where $n_{i, j}$ is the number of occurrences of the considered term in textual record $j$ and $\sum_{k} n_{k, j}$ is the sum of number of occurrences of all terms in textual record $j$.

Naturally, this procedure of keyword extraction is limited in its power to capture the contents of the text fields. The reason is that we are ignoring synonyms (which should be handled by one node rather than two or more), hyper- and hyponyms, pronouns (which may refer to a relevant keyword and thus may have to be counted for the occurrence of this keyword) etc. However, such linguistic properties are very difficult to take into account and need sophisticated tools (like thesauri etc.). Since such advanced text mining is not the main goal of our work (which rather focuses on BisoNet creation), keeping the processing simple seemed a feasible option. Nevertheless, advanced implementations may require such advanced processing, because ignoring, for example, synonyms and pronouns can distorts the statistics underlying, for instance, the term frequency value: ignoring pronouns that refer to a keyword, or not merging two synonyms makes the term frequency lower than it should actually be.

After all records have been processed, the inverse document frequency of each keyword $i$ is computed the following way: $\operatorname{idf}_{i}=\log \frac{|D|}{\left\{\left\{\in D \mid t_{i} \in d\right\} \mid\right.}$, where $|D|$ is the total number of records in the database and $\left|\left\{d \in D \mid t_{i} \in d\right\}\right|$ is the number of records in which the term $t_{i}$ appears.

Each node is then weighted with its corresponding average TFIDF value: tfidf $_{i}=\frac{1}{|D|} \sum_{j=1}^{|D|} \mathrm{tf}_{i, j} \cdot \mathrm{idf}_{i}$

This TFIDF approach is a very well known approach in text mining that is easy to implement and makes one able to easily apply a threshold, thus 
selecting only the most important nodes (keywords). A node then contains, as an attribute, a list of the term frequency values of its associated term in the different documents of the collection. This allows us to compute similarity measures presented in Section 4 in order to create links.

According to the definition of a bisociation presented in Section 2 , two concepts have to be linked by other concepts that are not in their proper domain (so-called bridging concepts). This leads us to introduce the notion of domains, into which the nodes are grouped, so that we can determine when borders between domains are crossed. In order to be able to classify nodes according to their membership in different domains, it is important that they keep, also as an attribute, the domains the data sources belong to, from which they have been extracted. Since the same keyword can occur in several data sources, taken from different domains, one has to be able (for example, for graph mining and link discovery purposes) to know whether a certain keyword has to be considered from a certain domain's point of view. The nodes therefore keep this information as vector of domains their associated keyword belongs to.

This can be interesting, for example, to mine or navigate the BisoNet, keeping in mind that a user may be looking for ideas related to a certain keyword belonging to a domain $A$. The results of a search for bisociations might also belong to domain $A$, because it is the domain of interest of the user. However, these results should be reached following paths using keywords from other domains, that is to say bisociations. This procedure provides related keywords of interest for the user, as they belong to its research domain, but they might be also original and new connections as they are the result of a bisociation process.

\section{Linking Nodes: Different Metrics}

As explained in Section 3.2 nodes are associated with a keyword and a set of documents in which this keyword occurs with a certain term frequency. Practically, this is represented using a vector of real values containing, for each document, the term frequency of the node's keyword. In order to determine whether a link should be created between two nodes or not, and if there is to be a link, to assign it a weight, we have to use a similarity measure to compare two nodes (that is to say: the two vectors of term frequency values).

Links in our BisoNets are weighted using similarity measures shown below. This approach allows us to use several different kinds of graph mining algorithms, such as simply thresholding the values to select a subset of the edges, or more complex ones, like calculating, for example, shortest paths.

\subsection{Cosine and Tanimoto Measures}

One basic metric that directly suggests itself is an adaptation of the Jaccard index (see [12]): $J(A, B)=\frac{|A \cap B|}{|A \cup B|}$.

Here $|A \cap B|$ represents the number of elements at the same index that both have a positive value in the two vectors and $|A \cup B|$ the total number of elements in the two vectors. 
It can also be interpreted as a probability, namely the probability that both elements are positive, given that at least one is positive (contain a given term $i$, i.e., $\left.\mathrm{tf}_{i}>0\right)$.

Cosine similarity is a measure of similarity between two vectors of $n$ dimensions by finding the angle between them. Given two vectors of attributes, $A$ and $B$, the cosine similarity, $\cos (\theta)$, is represented using a dot product and magnitude as $\cos (\theta)=\frac{A \cdot B}{\|A\|\|B\|}$, where, in the case of text matching, the attribute vectors $A$ and $B$ are usually the tf-idf vectors of the documents.

This cosine similarity metric may be extended such that it yields the Jaccard index in the case of binary attributes. This is the Tanimoto coefficient $T(A, B)$, represented as $T(A, B)=\frac{A \cdot B}{\|A\|^{2}+\|B\|^{2}-A \cdot B}$.

These measures allow us to compare two nodes according to the number of similar elements they contain, but do not take into account the importance of the text frequency values.

\subsection{The Bison Measure}

In the Jaccard measure, as applied above, we would consider only whether a term frequency is zero or positive and thus neglect the actual value (if it is positive). However, considering two elements at the same index $i$ in two vectors, one way of taking their values into account would be to use their absolute difference (that is, in our case, the absolute difference of the term frequency values for two terms, but the same document). With this approach, it is easy to compare two vectors (of term frequency values) by simply summing these values and dividing by the total number of values (or the total number of elements that are positive in at least one vector).

However, this procedure does not properly take into account that both values have to be strictly positive, because a vanishing term frequency value means that the two keywords do not co-occur in the corresponding document. In addition, we have to keep in mind that having two elements, both of which have a term frequency value of 0.2 , should be less important than having two elements with a term frequency value of 0.9 . In the first case, the keywords associated with the two nodes we are comparing appear only rarely in the considered document. On the other hand, in the latter case these keywords appear very frequently in this document, which means that they are strongly linked according to this document.

A possibility of taking the term frequency values itself (and not only their difference) into account is to use the product of the two term frequency values as a coefficient to the (absolute) difference between the term frequency values. This takes care of the fact that the two term frequency values have to be positive, and that the similarity value should be the greater, the larger the term frequency values are (and, of course, the smaller their absolute difference is). However, in our case, we also want to take into account that it is better to have two similar term frequency values of 0.35 (which means that the two keywords both appear rather infrequently in the document) than to have term frequency values of 0.3 and 0.7 (which means the first keywords appears rarely, while the other quite frequently). 
In order to adapt the product to this consideration, we use the expression in Equation 1, in which $k$ can be adjusted according to the importance one is willing to give to low term frequency values.

$$
B(A, B)=\left(\mathrm{tf}_{i}^{A} \cdot \mathrm{tf}_{i}^{B}\right)^{k} \cdot\left(1-\left|\mathrm{tf}_{i}^{A}-\mathrm{tf}_{i}^{B}\right|\right), \quad \mathrm{tf}_{i}^{A}, \mathrm{tf}_{i}^{B} \in[0,1]
$$

Still another thing that we have to take into account in our case is that the same difference between $\mathrm{tf}_{i}^{A}$ and $\mathrm{tf}_{i}^{B}$ can have a different impact depending on whether $\mathrm{tf}_{i}^{A}$ and $\mathrm{tf}_{i}^{B}$ are large or small. To tackle this issue, we combine Equation 1 with the use of the arctan function, and thus obtain the similarity measure shown in Equation 2, which we call the Bison measure. This form has the advantage that it takes into account that two term frequency values for the same index have to be positive, that the similarity should be the greater, the larger the term frequency values are, and that the same difference between $\mathrm{tf}_{i}^{A}$ and $\mathrm{tf}_{i}^{B}$ should have a different impact according to the values of $\mathrm{tf}_{i}^{A}$ and $\operatorname{tf}_{i}^{B}$.

$$
B(A, B)=\left(\mathrm{tf}_{i}^{A} \cdot \mathrm{tf}_{i}^{B}\right)^{k} \cdot\left(1-\frac{\left|\arctan \left(\mathrm{tf}_{i}^{A}\right)-\arctan \left(\mathrm{tf}_{i}^{B}\right)\right|}{\arctan (1)}\right), \quad \mathrm{tf}_{i}^{A}, \mathrm{tf}_{i}^{B} \in[0,1]
$$

\subsection{The Probabilistic Measure}

Another way of measuring the similarity between two nodes is based on a probabilistic view. Considering two terms, it is possible to compute, for each document they appear into, the probability of randomly selecting this document by randomly choosing an occurrence of the considered term, all of which are seen as equally likely. This value is given by the law of conditional probabilities shown in Equation 3

$$
\begin{array}{r}
P\left(d_{i} / t_{j}\right)=\frac{P\left(t_{j} / d_{i}\right) \cdot P\left(d_{i}\right)}{P\left(t_{j}\right)} \\
\text { with } P\left(t_{j}\right)=\sum_{d} P\left(t_{j} / d\right) \cdot P(d)
\end{array}
$$

This leads us to represent a node by a vector of all the conditional probabilities of the documents they appear in instead of a vector of text frequencies.

Having this representation, we can compare two nodes using the similarity measure shown in Equation 4 .

$$
S(A, B)=\sqrt{\frac{1}{n} \cdot \sum_{n}\left(P\left(d_{n} / t_{A}\right)-P\left(d_{n} / t_{B}\right)\right)^{2}}
$$

We can add that $P\left(d_{i} / t_{j}\right)$ in Equation 3 is equivalent to the term frequency if $P\left(d_{i}\right)$ is constant, which is the case in most of the textual data sources. We can however use this $P\left(d_{i}\right)$ to give arbitrary weights to certain documents. 


\section{Benchmarks}

Having shown how BisoNets can be built from textual data sources, we present benchmark applications in this section. The idea is to provide a proof of principle, that this approach of creating a BisoNet can help a user to discover bisociations.

In order to assess how effective the different similarity measures are, we count how many domain crossing links there are in the generated BisoNets, then we use different threshold values on the links in order to keep only the "strongest" edges according to the similarity measure used.

\subsection{The Swanson Benchmark}

Swanson's approach 13 to literature-based discovery of hidden relations between concepts $A$ and $C$ via intermediate $B$-terms is the following: if there is no known direct relation $A-C$, but there are published relations $A-B$ and $B-C$ one can hypothesize that there is a plausible, novel, yet unpublished indirect relation $A-C$. In this case the $B$-terms take the role of bridging concepts. In his paper [13, Swanson investigated plausible connections between migraine $(A)$ and magnesium $(C)$, based on the titles of papers published before 1987. He found eleven indirect relations (via bridging concepts $B$ ) suggesting that magnesium deficiency may be causing migraine.

We tried our approach on the Swansons data source which consists of 8000 paper titles, taken from the PubMed database, published before 1987 and talking about either migraine or magnesium, to see if it was possible to find again these relations between migraine and magnesium. In order to generate a BisoNet, we implemented a parser for text files containing the data from PubMed able to export them in the format understandable by the second layer of our framework. Then, this second layer performed the keywords extraction, using these keywords as nodes and linking these nodes in the way described in Section 3

By ranking and filtering the edges we then produced BisoNets that contained the "strongest" edges and their associated nodes. The left graphic of Figure 2 shows how many domain crossing links that are kept using different threshold values on the edges. On this graphic, we can observe that the Bison measure is the one able to keep the most crossing-domain links even if only the very strongest edges are kept (threshold set to keep only the best $5 \%$ of the edges). These tests demonstrate that the Bison measure is very well suited for bisociation discovery, since with it the strongest links are the bisociative ones.

We can observe this also in Figure 3 where the difference between the Tanimoto and the Bison measure is graphically highlighted, showing that if we keep only the $5 \%$ best edges, the Tanimoto measure loses any relation between magnesium and migraine whereas the Bison measure manages to keep at least some.

\subsection{The Biology and Music Benchmark}

As we aim to discover bisociations, that is associations between concepts that appear unrelated from a certain, habitual point of view, an interesting benchmark 

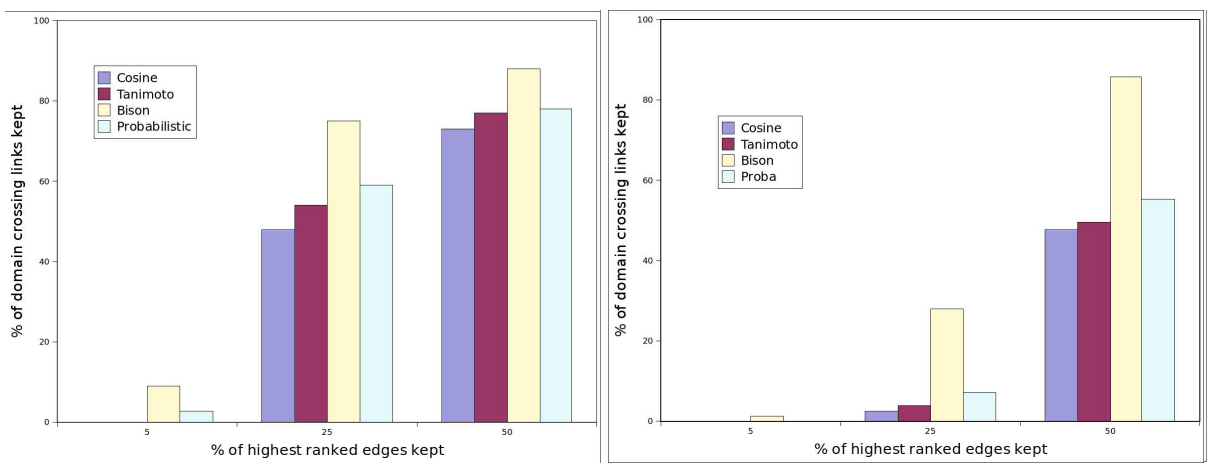

Fig. 2. Comparison between different similarity measures on the Swanson benchmark on the left and on the biology-music benchmark on the right

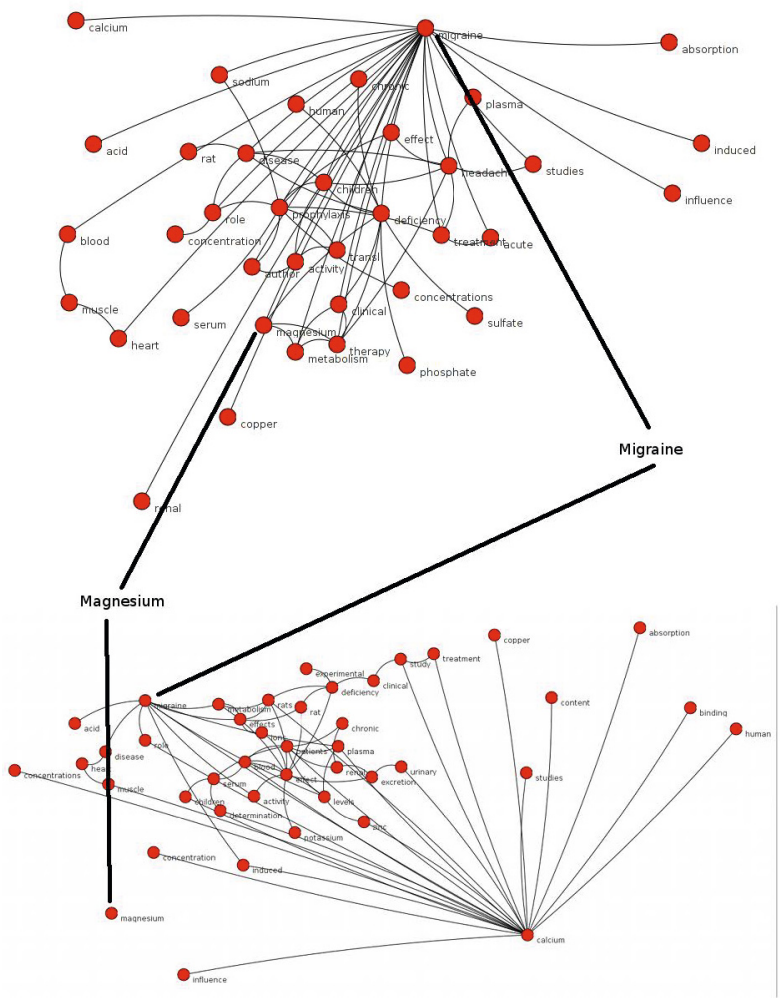

Fig. 3. Example of two BisoNets generated from the Swanson benchmark using the Bison similarity measure and the probabilistic similarity measure 
would be to look for bisociations in data coming from very different domains. We therefore use here data from two databases: the PubMed database that has already been talked about in the Swanson benchmark, and the FreeDB 3 database which is a freely available music database providing music titles, music styles and artist names.

We use exactly the same procedure as for the Swanson benchmark, that is reading the databases, performing textual pre-processing on terms and then launching the BisoNet creation framework to obtain a BisoNet containing terms linked to each other using the similarity distances described in this chapter. We consider here as potential keywords every word and author in the articles of the PubMed database, and every word of song titles, authors and styles in the FreeDB database.

The right graphic of Figure 2 shows how many domain crossing links that are kept using different threshold values on the edges.

\section{Conclusion}

In this chapter, we provided a definition of the notion of a bisociation, as understood by Koestler, which is the key notion of the BISON project. Building on this definition, we then defined the concept of a BisoNet, which is a network bringing together data sources from different domains, and therefore may help a user to discover bisociations. We presented a way we create nodes using simple text-mining techniques, and a procedure to generate links between nodes, which is based on comparing text frequency vectors using a new similarity measure.

We then tested our approach on benchmarks in order to rediscover bisociations between magnesium and migraine that have been discovered by Swanson using articles published before 1987. We see that bisociations between these two terms are easily discovered using the generated BisoNet, thus indicating that BisoNets are a promising technology for such investigations.

Using the second benchmark, we show that, even while mixing very different data sources, we are still able to produce BisoNets containing domain crossing links.

In summary, we venture to say that this work can be easily applied to any kind of textual data source in order to mine data looking for bisociations, thanks to the two layers architecture implementation.

Open Access. This article is distributed under the terms of the Creative Commons Attribution Noncommercial License which permits any noncommercial use, distribution, and reproduction in any medium, provided the original author(s) and source are credited.

\section{References}

1. Koestler, A.: The act of creation. London Hutchinson (1964)

2. Barron, F.: Putting creativity to work. In: The Nature of Creativity. Cambridge Univ. Press (1988)

\footnotetext{
3 http://www.freedb.org/
} 
3. Cormac, E.M.: A cognitive theory of metaphor. MIT Press (1985)

4. Agrawal, R., Imielinski, T., Swami, A.: Mining association rules between sets of items in large databases. In: Proceedings of the ACM SIGMOD Internation Conference on Management of Data, pp. 207-216 (1993)

5. Chalmers, D.J., French, R.M., Hofstadter, D.R.: High-level perception, representation and analogy: a critique of artificial intelligence methodology. Journal of Experimental and Theoretical Artificial Intelligence 4, 185-211 (1992)

6. Falkenhainer, B., Forbus, K.D., Gentner, D.: The structure mapping engine: algorithm and examples. Artificial Intelligence 41, 1-63 (1989)

7. Barnden, J.A.: An Implemented System for Metaphor-Based Reasoning - With Special Application to Reasoning about Agents. In: Nehaniv, C.L. (ed.) CMAA 1998. LNCS (LNAI), vol. 1562, pp. 143-153. Springer, Heidelberg (1999)

8. Aamodt, A., Plaza, E.: Case-based reasoning: foundational issues, methodological variations and system approaches. Artificial Intelligence Communications 7(1), 3959 (1994)

9. Cardoso, A., Costa, E., P.M.F.P.P.G.: An architecture for hybrid creative reasoning. In: Soft Computing in Case Based Reasoning. Springer, Heidelberg (2000)

10. van Rijsbergen, C.J., Robertson, S.E., Porter, M.F.: New models in probabilistic information retrieval. In: British Library Research and Development Report. Number 5587. London British Library (1980)

11. Gerard Salton, M.M.G.: Introduction to modern information retrieval. McGrawHill (1983)

12. Jaccard, P.: Étude comparative de la distribution florale dans une portion des alpes et du jura. Bulletin de la Société Vaudoise des Sciences Naturelles 37, 547-579 (1901)

13. Swanson, D.R., Smalheiser, N.R., Torvik, V.I.: Ranking indirect connections in literature-based discovery: The role of medical subject headings. Journal of the American Society for Information Science and Technology (JASIST) 57(11) (September 2006) 